\title{
Viscous Dissipation and Suction characteristics of Heat and Mass Transfer past a Stretching Sheet.
}

\author{
${ }^{1}$ AlfunsaPrathiba*, ${ }^{2}$ Dr.A.Venkata lakshmi \\ ${ }^{1}$ Department of Mathematics, CVR COLLEGE OF ENGINEERING \\ Hyderabad, India, alphonsaperli@gmail.com \\ ${ }^{2}$ Department of Mathematics, University College of Science, O.U \\ Hyderabad, India, akavaramvlr@gmail.com
}

Article History: Received: 11 January 2021; Accepted: 27 February 2021; Published online: 5 April 2021

\begin{abstract}
Soret number on heat and mass transfer Magneto Hydrodynamics (MHD) flow past an exponentially stretching sheet with the heat source/sink. Appropriate similarity transformations were employed to convert the governing partial differential equations to a set of highly non-linear ordinary differential equations, which was then solved numerically by Runga kutta sixth order method together with shooting technique. The Numerical results are obtained for the skin friction coefficient, Nusselt and Sherwood numbers for selected values of the governing parameters, such as the suction, magnetic field parameter, viscous dissipation parameter, heat generation parameter , Schmidt number , and the chemical reaction rate parameter. Besides, it is obtained that the concentration profile decreases with an increment of the Schmidt number. A comparison was made with a previous study available in the literature and we found that it is in a good agreement.
\end{abstract}

Keywords- Magneto Hydrodynamics (MHD), Boundary layer flow, Suction, stretching sheet

\section{INTRODUCTION}

Magneto Hydrodynamics (MHD) boundary layer flow of heat and mass transmit over a stretching sheet has wide applications in industrial and manufacturing process. Some of applications are hot rolling, wire drawing, glass-fiber, and paper production, drawing of plastic films, metal and polymer extraction, and metal spinning. In the process of manufacturing the following activities like simultaneous heating or cooling and kinematics of stretching has a decisive influence on the quantity of the final products (Magayari and Keller [1]). The first person who investigated the similarity solution for laminar boundary layer flow and the transfer of heat over a stretching surface was Crane [2]. Later, many researchers have studied on the stretching sheet problems and presented a detailed analysis by including different aspects, such as heat flux, permeability and unsteadiness characteristics, etc. Carragher and Crane [3], Dutta [4], Grubka and Bobba [5], Elbassebeshy [6], Elbashabeshy and Bazid [7], Mahapatra and Gupta [8] are among the researchers who studied on it. Recently Mukhopahyay [9] presented heat transfer and Magneto Hydrodynamics (MHD) boundary layer flow towards an exponentially stretching sheet embedded in a thermally stratified medium subject to suction. He has shown that increasing the magnetic parameter reduces the fluid velocity. Viscous dissipation has extensive industrial applications: for example, a considerable temperature rises are observed in polymer processing flows such as injection modeling or extraction at high rates. Aerodynamic heating in the thin boundary layer around high-speed aircraft raises the temperature of the skin. The processes of converting mechanical energy of downward flowing water into thermal and acoustical energy are dissipation. Jena et al. [10] considered the diffusion-thermo (Dufour) and thermal diffusion (Soret) impact on MHD visco-elastic fluid flow over a porous vertical stretching sheet subject to variable magnetic field embedded in a porous medium in the attendance of chemical reaction and heat source/sink.

Seini and Makinde [11] have examined and MHD boundary layer flow of a viscous incompressible steady fluid over an exponentially stretching sheet with the impact of a homogeneous chemical reaction and radiation. Their result indicated that raising the values of the transverse magnetic field and radiation parameter reduce heat transfer rate at the surfaces. Heat and mass transfer on a boundary layer of an electrically conducting viscous fluid through a porous media over an exponentially stretching sheet with an impact of a magnetic field was investigated by Swain et al. [12]. In their study, they also considered the effect heat source/sink and thermal radiation. In the context of exothermic and endothermic chemical reactions, heat generation is very valuable. Mass transfer effect on MHD flow past an impulsively started infinite vertical plate was presented by Shankar and Kishan [13]. Furthermore, flow on an MHD boundary layer, heat and mass transfer of an incompressible viscous and radiating fluid due to an exponentially stretching sheet were studied by Devi et al. [14].

An MHD flow on a boundary layer and the characteristics of heat transfer of a non-Newtonian visco-elastic fluid over a flat plate with a linear velocity in the attendance of non-uniform heat source and thermal radiation were inspected by Abel and Mahesha [15]. In addition, the MHD flow of a Newtonian liquid and the properties 
of heat and mass transfer over an exponentially stretching sheet with radiation effect have been examined by Kameswaran et al. [16]. Their result showed that the Prandtl number and radiation parameter have an inverse effect on temperature profile. Moreover, Khalili et al. [17] examined MHD boundary layer flow past an exponentially stretching with chemical reaction, radiation and heat sink. They have observed that the reaction rate parameter affected the concentration profiles significantly and the concentration thickness of boundary layer decreases when the reaction rate parameter increases.

A vast body of knowledge encompassing analytical and numerical studies explaining various aspects is now available on the stretching flow [18]-[27]. To the best of the author's knowledge, the effect of chemical reaction and viscous dissipation on MHD flow past an exponentially stretching sheet with a heat sink is not studied adequately in a comprehensive way. Hence, this problem is investigated. The aim of this investigation is to discuss such a flow problem. Similarity variables have been used to transform the governing partial differential equations into nonlinear ordinary differential equations. So as to reveal the impact of various governing parameters on the velocity, a temperature, concentration, coefficient of skin friction, Nusselt number and Sherwood number a parametric analysis is accomplished and discussed in detail.

\section{MATHEMATICAL FORMULATION}

In this article, a laminar two dimensional steady flow of an incompressible viscous, electrically conducting fluid over a continuous exponentially stretching surface is considered. The origin of the system is positioned at the slit from which the sheet is drawn. In this the frame of the $x$-axis is taken along the path of the continuous stretching plane.

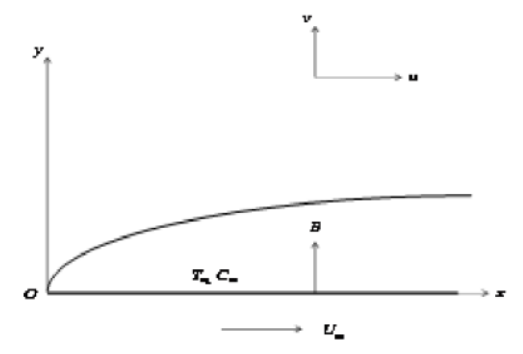

Figure .1 Schematics of the problem.

The $y$ - axis is measured normal to the surface of the sheet. The sheet velocity supposed to vary as an exponential function of the distance $\mathrm{x}$ from the slit. The temperature and concentration far away from the fluid as displayed in Figure 1 are denoted by respectively. The concentration differences and ambient temperature sheet are also magnetic field $T_{\infty}$ and $C_{\infty}$ of strength pertained normal to the sheet is denoted by. The variable chemical reaction is imagined to be $k_{l}(x)$ and variable heat sink parameter $Q^{*}(x)$, where $k_{0}$ and $Q_{0}$ are constants. The parameters $T_{w}, C_{w}$, and $B_{0}$ stand for the temperature at the surface of the sheet, concentration at the surface of the sheet and $B(x)$ magnetic field strength respectively.

Under above $B(x)$ assumptions, the governing equation of the momentum, heat and mass transfer transports subject to viscous dissipation and heat generation can be written as:

$$
\begin{gathered}
\frac{\partial u}{\partial x}+\frac{\partial v}{\partial y}=0 \\
u \frac{\partial u}{\partial x}+v \frac{\partial u}{\partial y}=v \frac{\partial^{2} \mathrm{u}}{\partial \mathrm{y}^{2}}-\frac{\sigma \mathrm{B}^{2}(\mathrm{x})}{\rho} \mathrm{u} \\
u \frac{\partial T}{\partial x}+v \frac{\partial T}{\partial y}=\frac{k}{\rho c_{p}} \frac{\partial^{2} T}{\partial y^{2}}+\frac{Q^{*}(x)}{\rho c_{p}}\left(T-T_{\infty}\right)+\frac{\mu}{\rho c_{p}}\left(\frac{\partial u}{\partial y}\right)^{2} \\
u \frac{\partial C}{\partial x}+v \frac{\partial C}{\partial y}=D \frac{\partial^{2} C}{\partial y^{2}}-k_{1}\left(C-C_{\infty}\right)
\end{gathered}
$$

The velocity components in $x$ and $y$ direction are symbolized by $u$ and $v \square \square$ respectively. The kinematic viscosity $v$, the fluid density $\rho$, the temperature of the fluid $T$, the thermal conductivity $k$, the specific heat at 
constant temperature $C_{p}$, the boundary layer fluid concentration $C$, the mass diffusivity $D$, and the rate of chemical reaction parameter is represented by $k_{l}$.

The boundary conditions associated for the velocity, temperature, and concentration profiles are

$$
\begin{aligned}
& \left.\begin{array}{r}
u=U_{\omega}=U_{0} e^{\frac{x}{L}}, v=-V_{\omega}(x) \\
T=T_{\omega}=T_{\infty}+T_{0} e^{\frac{x}{2 L}} \\
C=C_{\omega}=C_{\infty}+C_{0} e^{\frac{x}{2 L}}
\end{array}\right\} \text { at } y=0 \\
& u \rightarrow 0, T \rightarrow T_{\infty}, C \rightarrow C_{\infty} \text {, as } y \rightarrow \infty
\end{aligned}
$$

Where $\boldsymbol{U}_{w}$ is the uniform velocity of the sheet and $L$ is the reference length. Introducing the following dimensionless quantities the mathematical analysis of the problem is simplified as

$$
\begin{gathered}
\eta=\sqrt{\frac{U_{0}}{2 v L}} e^{\frac{x}{2 L}} y, u=U_{0} e^{\frac{x}{L}} f^{\prime}(\eta) \\
v=-\sqrt{\frac{v U_{0}}{2 L}} e^{\frac{x}{2 L}}\left(f(\eta)+\eta f^{\prime}(\eta)\right) \\
T=T_{\infty}+T_{0} e^{\frac{x}{2 L}} \theta(\eta), \\
C=C_{\infty}+C_{0} e^{\frac{x}{2 L}} \phi(\eta), \\
B(x)=B_{0} e^{\frac{x}{2 L}}, \\
K_{p}(x)=K_{0} e^{\frac{x}{L}} \\
Q^{*}(x)=Q_{0} e^{\frac{x}{2 L}} \\
V_{w}(x)=V_{0} e^{\frac{x}{2 L}} .
\end{gathered}
$$

Where $\eta$ is the similarity variable, $f(\eta)$ is the dimensionless stream function, $\theta(\eta)$ is the dimensionless temperature and $\phi(\eta)$ is the dimensionless concentration, $\mathrm{u}$ and $\mathrm{v}$ are also defined using the stream function as:

$$
u=\frac{\partial \psi}{\partial y} \text { and } v=-\frac{\partial \psi}{\partial x}
$$

Which satisfy equation (1).Then by using the similarity transformation, the governing equations (2)-(4) are transformed into ordinary differential equations as follows :

$$
\begin{array}{r}
f^{\prime \prime \prime}+f f^{\prime \prime}-2 f^{\prime 2}-M f^{\prime}=0 \\
\theta^{\prime \prime}+\operatorname{Pr}\left(f \theta^{\prime}-f^{\prime} \theta+\delta \theta+E c f^{\prime 2}\right)=0 \\
\phi^{\prime \prime}+S c\left(f \phi^{\prime}-f^{\prime} \phi-k 1 \phi\right)=0
\end{array}
$$

With the boundary conditions as:

$$
\left.\begin{array}{c}
f(0)=S, f^{\prime}(0)=1, \theta(0)=1, \phi(0)=1 \text { at } \eta=0 \\
f^{\prime}(\eta)=0, \theta(\eta)=0, \phi(\eta)=0 \text { as } \eta \rightarrow \infty
\end{array}\right\}
$$

The parameters involved in the above equations are the Eckert number $\boldsymbol{E} \boldsymbol{c}$, magnetic parameter $M$, Prandtl number $P r$, heat generation $\delta$, Schmidt number $S c$, chemical reaction rate parameter $k_{l}$, and the Suction parameter $S$. These parameters are defined by: 


$$
\left.\begin{array}{c}
S=\frac{V_{0}}{\sqrt{\frac{v_{0}}{2 L}}}, M=\frac{2 \sigma B_{0}{ }^{2} L}{\rho U_{0}} \\
E c=\frac{U_{\omega}{ }^{2}}{c_{\rho}\left(T_{\omega}-T_{\infty}\right)}, \delta=\frac{2 L Q_{0}}{\rho c_{p} U_{0}} \\
k 1=\frac{2 k_{0} L}{U_{0}} \\
S c=\frac{v}{D}
\end{array}\right\}
$$

The physical quantities which are involved are the skin friction coefficient, the local Nusselt number, and the Sherwood number . These quantities can be defined as

$$
\begin{gathered}
C_{f}=\frac{2 \tau_{\omega}}{\rho U_{\omega}{ }^{2}} \\
N u=\frac{x q_{\omega}}{k\left(T_{\omega}-T_{\infty}\right)} \\
S h=\frac{x j_{\omega}}{D\left(C_{\omega}-C_{\infty}\right)}
\end{gathered}
$$

Where $\tau_{w}, q_{w} j_{w}$ are the shear stress, heat flux, and mass flux at the surface respectively and they are defined by:

$$
\begin{aligned}
& \tau_{\omega}=-\mu\left(\frac{\partial u}{\partial y}\right)_{y=0}=\frac{-\mu U_{0}}{L} \sqrt{\frac{R \theta}{2}} e^{\frac{3 x}{2 L}} f^{\prime \prime}(0) \\
& q_{\omega}=-k\left(\frac{\partial T}{\partial y}\right)_{y=0}=\frac{-k\left(T_{\omega}-T_{\infty}\right.}{L} \sqrt{\frac{R \theta}{2}} e^{\frac{x}{2 L}} \theta^{\prime}(0) \\
& j_{\omega}=-D\left(\frac{\partial C}{\partial y}\right)_{y=0}=\frac{-D\left(c_{\omega}-c_{\infty}\right.}{L} \sqrt{\frac{R \varepsilon}{2}} e^{\frac{x}{2 L}} \phi^{\prime}(0)
\end{aligned}
$$

Using equations (16)-(18), equations (13) - (15) can be transformed into

$$
\begin{aligned}
& \frac{\sqrt{\frac{R e}{2}} c_{f}}{\sqrt{\frac{x}{L}}}=-f^{\prime \prime}(0) \\
& \frac{N u}{\sqrt{\frac{x}{L}} \sqrt{\frac{R e}{2}}}=-\theta^{\prime}(0) \\
& \frac{S h}{\sqrt{\frac{x}{L}} \sqrt{\frac{R e}{2}}}=-\phi^{\prime}(0)
\end{aligned}
$$

Where $\mu$ is the coefficient of viscosity and $\operatorname{Re}=\frac{U_{0} L}{v}$ is the Reynolds number.

\section{SOLUTION}

The transformed ordinary differential equations (8)-(10) along with the boundary conditions (11) are solved using Mathematica package with inbuilt Runge Kutta Sixth order method using NDSolve command. The 
supposed step size 0.01 and the accuracy convergence were set to five decimal values before solving the problem using Mathematica.

\section{RESUlTS AND DissCUSION}

The transformed ordinary equations of momentum, energy, and concentration (8)-(10) subjected to the boundary conditions were numerically solved by using Runge Kutta sixth order method using NDsolve algorithm in Mathematica package. We obtained the graph of velocity, temperature, and concentration profile for diverse values of governing parameters. The results obtained are displayed through tables and figures. The coefficient of skin friction for various values of the magnetic parameter and for a fixed values of $S=E c=P r=\delta=k_{l}=S c=0$ is displayed in Table 1. We have observed that an increase of magnetic parameter M raises the skin friction coefficient; as a result of the opposition to the flow caused by the induced Lorentz force. The present result is in a good agreement with the previously published results.

TABLE I. A COMPARISON OF THE SKIN FRICTION COEFFICIENT FOR DIFFERENT VALUES OF M AND FOR $S=E C=P r=\delta=K_{l}=S C=0$ $-f^{\prime \prime}(0)$

\begin{tabular}{|c|c|c|c|}
\hline \multirow{2}{*}{$\mathbf{M}$} & \multicolumn{3}{|c|}{$-f^{\prime \prime}(0)$} \\
\cline { 2 - 4 } & Kameswaran et al. [16] & $\begin{array}{c}\text { Sai et } \\
\text { al. } \\
{[28]}\end{array}$ & Present \\
\hline 0 & 1.28181 & 1.29038 & $\begin{array}{c}1.28186 \\
1\end{array}$ \\
\hline 1 & 1.62918 & 1.63038 & $\begin{array}{c}1.62919 \\
0\end{array}$ \\
\hline 2 & 1.91262 & 1.91285 & $\begin{array}{c}1.91262 \\
5\end{array}$ \\
\hline 3 & 2.15874 & 2.15879 & 2.15874 \\
& & & 3 \\
\hline 4 & 2.37937 & 2.37938 & 2.37938 \\
& & & \\
\hline
\end{tabular}

Fig. 2 displays the suction parameter consequence on the velocity profile. We observed that the velocity profile decreases with an increase in the suction parameter. The impact of the suction parameter on the temperature profile is illustrated by Fig. 3. It is watched that the temperature profile is reduced with an enhancement of the suction parameter. The thickness of the thermal boundary layer lessened with an increase of the suction parameter. The influence of the suction parameter on the concentration profile is displayed using Fig. 4. Thus, we observed that an increment o the suction parameter S reduces the concentration profile. Fig. 5 shows the influence of a viscous dissipation parameter on the temperature field. It is seen that the thickness of the thermal boundary layer higher with an enlargement of viscous dissipation parameter . Fig. 6 exemplifies the heat generation parameter influence on the concentration profile. It is watched that the heat generation parameter reduces the concentration profile. The Schmidt number and chemical reaction impact on the concentration profile is shown by Fig. 7 and Fig. 8, respectively. The result has displayed that, an increment of both parameters reduces the concentration profile. In the case of Schmidt number, increasing the size of means decreasing the diffusivity of the fluid, this causes the fluid less concentrated. The influences of magnetic field parameter on the velocity field and temperature profile are shown by Fig. 09 and Fig. 10, respectively. It is obtained the temperature profile increases while the velocity profile decreases with an increment of . Since a Lorentz force is created due to the occurrence of that slows down the motion of the fluid, as a result, the distribution of velocity is reduced and the thermal boundary layer thickness is increased.

\section{CONCLuSION}

In this article, the effect of different flow parameters on the dimensionless velocity profile, temperature profile, the concentration profiles were considered. The governing problem was solved numerically using RK sixth order method along with shooting technique. It is observed that:

(i) Increment of has a reducing effect on the velocity, temperature and concentration profiles.

(ii) An increase of the viscous dissipation parameter increases the temperature profile,

(iii) Increasing the heat generation parameter enhances the temperature profile.

(iv) As the values of and increases the concentration profile decreases.

\section{References.}


1. M. E and K. B, "Heat and mass transfer in the boundary layers on an exponentially stretching continuous surface,” J. Phys. DAppl.Phys, vol. 32, pp. 577-585, 1999.

2. L. Crane, "Flow past a stretching plate," Zeitschrift fur Angew. Mathe-matik und Phys. ZAMP, vol.21, no. 4, pp. 645-647, 1970.

3. P.Carragher and L.Crane, "Heat transfer on a continuous stretching sheet," Z.Angew.Math. Mech,vol. 62, no. 10, pp. 564-565, 1982.

4. B. Dutta, P. Roy, and A. . Gupta, "Temperature field in the flow over a stretching sheet with uniform heat flux,” Int. J Commun. Heat.MassTransf, vol. 12, pp. 89-94, 1985.

5. L.Grubka and K. . Bobba, "Heat transfer characteristics of a continuous stretching surface with variable temperature," ASME J. Heat Transf., vol. 107, 1985.

6. E. M. Elbashbeshy, "Heat transfer over a stretching surface with variable surface heat flux,"J.Phys.D:Appl.Phys, vol. 31, no. 16, p. 1951-, 1998.

7. E.Elbashbeshy and M.Bazid, "Heat transfer over an unsteady stretching surface," HeatMassTransf, vol. 41, no. 1, pp. 1-4, 2004.

8. T.R.Mahapatra and A.Gupta, "Heat transfer in stagnation-point flow towards a stretching sheet," Heat MassTransf, vol. 38, no. 6, pp. 517-521, 2002.

9. S. Mukhopadhyay, "MHD boundary layer flow and heat transfer over an exponentially stretching sheet embedded in a thermally stratified medium," Alexandria Eng. J., vol. 52, pp. 259-265, 2013.

10. S. Jena, G. C. Dash, and S. R. Mishra, "Chemical reaction effect on MHD viscoelastic fluid flow over a vertical stretching sheet with heat source/sink," Ain Shams Eng. J., 2016.

11. Y. I. Seini and O. D. Makinde, "MHD Boundary Layer Flow due to Exponential Stretching Surface with Radiation and Chemical Reaction," Hindawi Publ. Corp. Math. Probl. Eng., 2013.

12. Swain, S. R. Mishra, and H. B. Pattanayak, "Flow over Exponentially Stretching Sheet through Porous Medium with Heat Source/Sink," Hindawi Publ. Corp. J. Eng., 2015.

13. B. Shanker and N. Kishan, "The effects of mass transfer on the MHD flow past an impulsively started infinite vertical plate with variable temperature or constant heat flux," J. Eng. Heat Mass Transf., vol. 19, pp. 273-278, 1997.

14. R. L. V. R. Devi, T. Poornima, N. B. Reddy, and S. Venkataramana, "Radiation and Mass Transfer Effects on MHD Boundary Layer Flow due to an Exponentially Stretching Sheet with Heat Source," Int. J. Eng. Innov. Technol., vol. 3, no. 8, 2014.

15. M. Subhas Abel and N. Mahesha, "Heat transfer in MHD viscoelastic fluid flow over a stretching sheet with variable thermal conductivity, non-uniform heat source and radiation," Appl. Math. Model., vol. 32, pp. 1965-1983, 2008.

16. P. K. Kameswaran, M. Narayana, P. Sibanda, and G.Makanda, "On radiation effects on hydromagnetic Newtonian liquid flow due to an exponential stretching sheet," Bound. Value Problem, vol. 105, pp. 1$16,2012$.

17. N. N. W. Samson, K. A. Aziz, A. S. A. Aziz, and Z. M. Ali, "Chemical reaction and radiation effects on MHD flow past an exponentially stretching sheet with heat sink," IOP Conf. Ser. J. Phys., vol.Conf. Seri, 2017.

18. R. Cortell, "Effects of viscous dissipation and work done by deformation on the MHD flow and heat transfer of a viscoelastic fluid over a stretching sheet," Phys. Lett., vol. 357, pp. 298-305,2006.

19. R. Cortell, "A note on flow and heat transfer of a viscoelastic fluid over a stretching sheet," Int. J.NonLinear Mech, vol. 41, pp. 78-85, 2006.

20. K. Sadeghy, A. H. Najafi, and M. Saffaripour, "Sakiadis flow of an upper-convected Maxwell fluid," Int. J. Non-Linear Mech, vol. 40, pp. 1220-1228, 2005.

21. C. Liu, "Flow and heat transfer of an electrically conducting fluid of second grade in a porous medium over a stretching sheet subject to a transverse magnetic field," Int. J. Non-Linear Mech,vol. 40, pp. 465-474, 2005.

22. P. D. Ariel, T. Hayat, and S. Asghar, "The flow of an elastic-viscous fluid past a stretching sheet with partial slip," Acta Mech, vol. 187, pp. 29-35, 2006.

23. S. J. Liao and A. Campo, "Analytic solutions of the temperature distribution in Blasius viscous flow problems," J. Fluid Mech, vol. 453, pp. 411-425, 2002.

24. S. J. Liao, "On the analytic solution of magnetohydrodynamic flows of non-Newtonian fluid over a stretching sheet," J. Fluid Mech, vol. 488, pp. 189-212, 2003.

25. H. Xu and S. J. Liao, "Series solutions of unsteady magnetohydrodynamic flows of non-Newtonian fluids caused by an impulsively stretching plate," J. Non-Newton. Fluid Mech, vol. 129, pp. 46-55, 2005.

26. T. Hayat, Z. Abbas, and M. Sajid, "Series solution for the upper-convected Maxwell fluid over a porous stretching plate,” Phys. Lett, vol. 358, pp. 396-403, 2006. 
27. T. Hayat and M. Sajid, "Analytic solution for axisymmetric flow and heat transfer of a second grade fluid past a stretching sheet," Int. J. Heat Mass Transf., vol. 50, pp. 75-84, 2007.

28. P. S. Sai, K. Ramakrishna, N. Puppala, and K. J. Reddy, "chemical reaction and radiation effects on mhd flow over an exponentially stretching sheet with viscous dissipation and heat generation," Int. J. Math. Comput. Appl. Res., vol. 5, no. 3, pp. 35-48, 2015.

29. R. R. Devi, T. Poornima, B. R. N, and V. S, "Radiation and mass transfer effects on MHD boundary layer flow due to an exponentially stretching sheet with heat source," IJIET, vol. 3, no. 8, pp. 33-39, 2014.

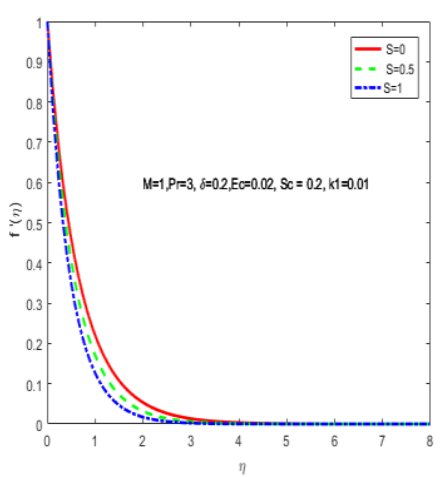

Fig. 2: The influence of $S$ Suction parameter on the velocity field.

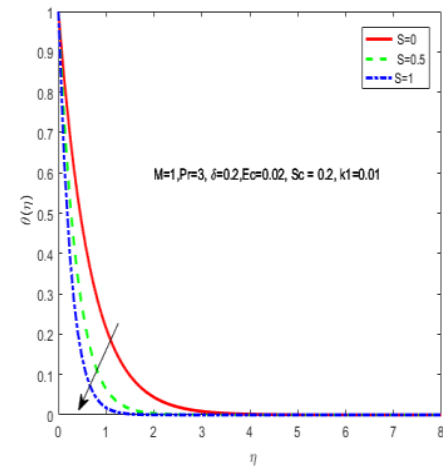

Fig. 3: The influence of $S$ Suction parameter on the temperature field.

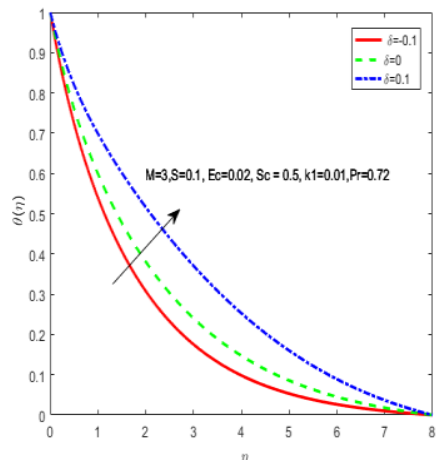

Fig. 6 Temperature profile with a change of $\hat{\sigma}_{\text {. }}$

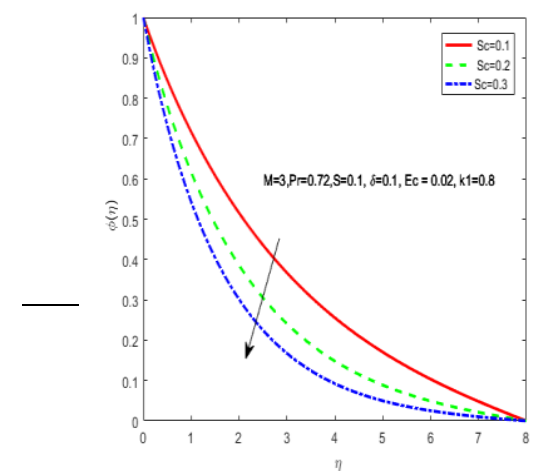

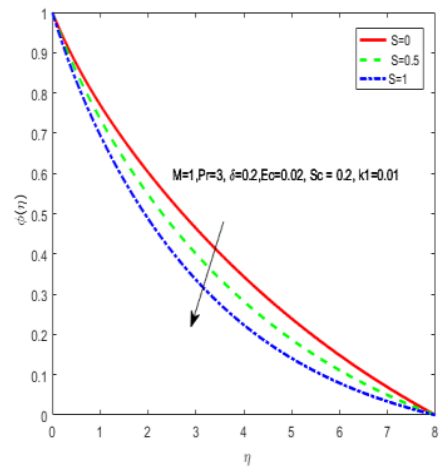

Fig. 4: The influence of $S$ Suction parameter on the concentration distribution.

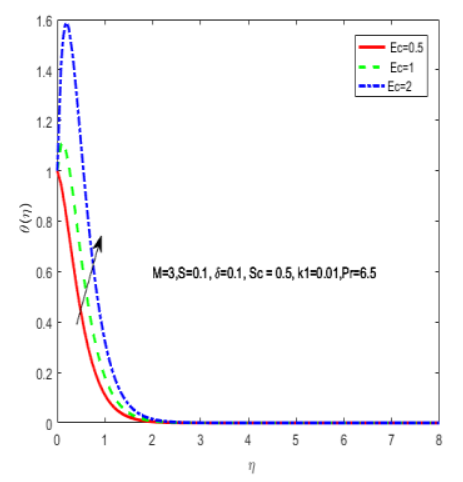

Fig. 5: Temperature profile with an effect of Eckert number Ec.

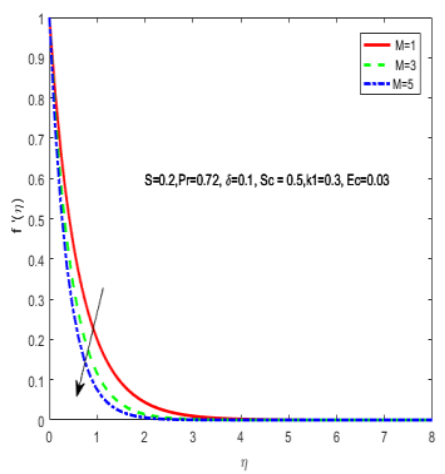

Fig. 9: The behavior of velocity profile with a variation of Magnetic parameter $M$

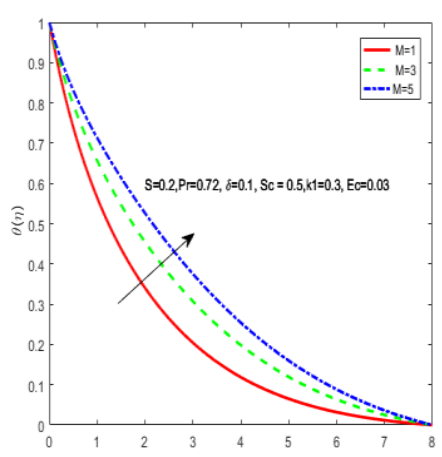


Fig.7: The impact of Se Schmidt number on the concentration profile temperature.

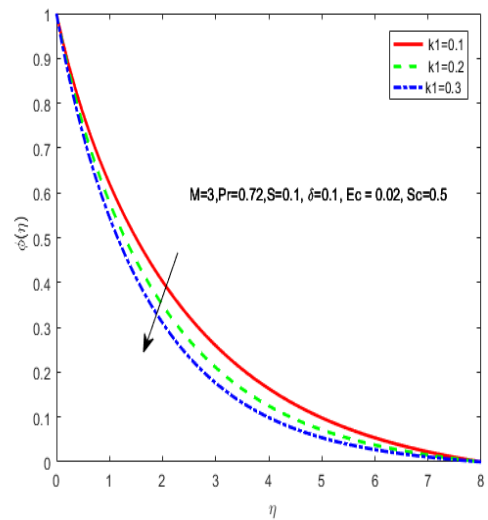

Fig.8: The impact of $K 1$ chemical reaction parameter on the concentration profile.
Fig. 10: The impact of $M$ Magnetic parameter on the 